\title{
Models within Models: Taming Model Complexity Using the Sub-model Lattice
}

\author{
Pierre Kelsen, Qin Ma, and Christian Glodt \\ Laboratory for Advanced Software Systems \\ University of Luxembourg \\ 6, rue Richard Coudenhove-Kalergi \\ L-1359 Luxembourg \\ \{Pierre.Kelsen, Qin.Ma, Christian.Glodt\}@uni.lu
}

\begin{abstract}
Model-driven software development aims at easing the process of software development by using models as primary artifacts. Although less complex than the real systems they are based on, models tend to be complex nevertheless, thus making the task of comprehending them non-trivial in many cases. In this paper we propose a technique for model comprehension based on decomposing models into sub-models that conform to the same metamodel as the original model. The main contributions of this paper are: a mathematical description of the structure of these sub-models as a lattice, a linear-time algorithm for constructing this decomposition and finally an application of our decomposition technique to model comprehension.
\end{abstract}

\section{Introduction}

In model-driven software development models are the primary artifacts. Typically several models are used to describe the different concerns of a system. One of the main motivations for using models is the problem of dealing with the complexity of real systems: because models represent abstractions of a system, they are typically less complex than the systems they represent.

Nevertheless models for real systems can be complex themselves and thus may require aids for facilitating human comprehension. The problem of understanding complex models is at the heart of this paper. We propose a method for decomposing models that is based on subdividing models into smaller sub-models with the property that these sub-models conform to the same metamodel as the original model. This property allows to view the sub-models using the same tools as the original model and to understand the meaning of the sub-models using the same semantic mapping (if one has been defined).

An example of a concrete application scenario is the following: when trying to understand a large model, one starts with a subset of concepts that one is interested in (such as the concept of Class in the UML metamodel). Our method allows to construct a small sub-model of the initial model that contains all entities of interest and that conforms to the original metamodel (in the case of UML this would be MOF). The latter condition ensures that the sub-model can

D. Giannakopoulou and F. Orejas (Eds.): FASE 2011, LNCS 6603, pp. 171 185, 2011.

(C) Springer-Verlag Berlin Heidelberg 2011 
be viewed in the same way as the original model and that it has a well-defined semantics. The smaller size (compared to the original model) should facilitate comprehension.

The main contributions of this paper are the following: (a) we present the mathematical structure of these sub-models as a lattice, with the original model at the top and the empty sub-model at the bottom; (b) we present a linear time algorithm for building a decomposition hierarchy for a model from which the sub-model lattice can be constructed in a straightforward manner; (c) we present a method for the user to comprehend models in the context of model pruning.

One salient feature of our technique is its generic nature: it applies to any model (even outside the realm of model-driven software development). This is also one of the main features differentiating it from existing work in model decomposition. We review here related work from model slicing, metamodel pruning and model abstraction.

The idea behind model slicing is to generalize the work on program slicing to the domains of models by computing parts of models that contain modeling elements of interest. An example of this line of work is [4] where model slicing of UML class diagrams is investigated. Another example is [2 which considers the problem of slicing the UML metamodel into metamodels corresponding to the different diagram types in UML. The main differences between our work and research on model slicing is, first, the restriction of model slicing to a particular modeling language, e.g. UML class diagrams, and, second, the focus on a single model rather than the mathematical structure of sub-models of interest.

In a similar line of work some authors have investigated the possibility of pruning metamodels in order to make them more manageable. The idea is to remove elements from a metamodel to obtain a minimal set of modeling elements containing a given subset of elements of interest. Such an approach is described in [8]. This work differs from our work in several respects: first, just like model slicing it focuses on a single model rather than considering the collection of relevant sub-models in its totality; second, it is less generic in the sense that it restricts its attention to Ecore metamodels (and the pruning algorithm they present is very dependent on the structure of Ecore), and lastly their goal is not just to get a conformant sub-model but rather to find a sub-metamodel that is a supertype of the original model. This added constraint is due to main use of the sub-metamodel in model transformation testing.

The general idea of simplifying models (which can be seen as a generalization of model slicing and pruning) has also been investigated in the area of model abstraction (see [3] for an overview). In the area of simulation model abstraction is a method for reducing the complexity of a simulation model while maintaining the validity of the simulation results with respect to the question that the simulation is being used to address. Work in this area differs from ours in two ways: first, model abstraction techniques generally transform models and do not necessarily result in sub-models; second, conformance of the resulting model with a metamodel is not the main concern but rather validity of simulation results. 
The remainder of this paper is structured as follows: in the next section we present formal definitions for models, metamodels and model conformance. In section 3 we describe an algorithm for decomposing a model into sub-models conformant to the same metamodel as the original model. We also present the mathematical structure of these models, namely the lattice of sub-models. In section 4 we outline the application to model comprehension and we present concluding remarks in the final section.

\section{Models and MetaModels}

In this section we present formal definitions of models, metamodels, and model conformance. The following notational conventions will be used:

1. For any tuple $p$, we use fst $(p)$ to denote its first element, and snd $(p)$ to denote its second element.

2. For any set $s$, we use $\sharp s$ to denote its cardinality.

3 . We use $\leq$ to denote the inheritance based subtyping relation.

\section{$2.1 \quad$ Metamodels}

A metamodel defines (the abstract syntax of) a language for expressing models. It consists of a finite set of metaclasses and a finite set of relations between metaclasses - either associations or inheritance relations. Moreover, a set of constraints may be specified in the contexts of metaclasses as additional well-formedness rules.

Definition 1 (Metamodel). A metamodel $\mathbb{M}=(\mathbb{N}, \mathbb{A}, \mathbb{H}, \mathbb{C})$ is a tuple:

$-\mathbb{N}$ is the set of metaclasses, and $\mathrm{n} \in \mathbb{N}$ ranges over it.

- $\mathbb{A} \subseteq(\mathbb{N} \times \mu) \times(\mathbb{N} \times \mu \times \mathbb{R})$ represents the (directed) associations between metaclasses and $\mathbb{a} \in \mathbb{A}$ ranges over it. The two $\mathbb{N}$ 's give the types of the two association ends. The two $\mu$ 's, where $\mu \in \operatorname{Int} \times\{\operatorname{Int} \cup\{\infty\}\}$, give the corresponding multiplicities. We refer to the first end of the association as the source, and the second as the target. Associations are navigable from source to target, and the navigation is represented by referring to the given role name that is attached to the target end selected from the vocabulary $\mathbb{R}$ of role names.

$-\mathbb{H} \subseteq \mathbb{N} \times \mathbb{N}$ denotes the inheritance relation among metaclasses and $\mathbb{h} \in \mathbb{H}$ ranges over it. For a given $\mathbb{h} \in \mathbb{H}$, fst(h) inherits from (i.e., is a subtype of) snd (h) .

- $\mathbb{C} \subseteq \mathbb{N} \times \mathbb{E}$ gives the set of constraints applied to the metamodel and $\mathbb{C} \in \mathbb{C}$ ranges over it. $\mathbb{E}$ is the set of the expressions and $\mathbb{E} \in \mathbb{E}$ ranges over it. $A$ constraint $\mathbb{C}=(\mathrm{m}, \mathbb{e})$ makes the context metaclass $\mathrm{m}$ more precise by restricting it with an assertion, i.e., the boolean typed expression $\mathbb{e}$. For example, a constraint can further restrict the multiplicities or types of association ends that are related to the context metaclass.

Let $r \in \mathbb{R}$ range over the set of role names mentioned above. We require that role names in a metamodel are distinct. As a consequence, it is always possible to retrieve the association that corresponds to a given role name, written asso(r). 


\subsection{Models}

A model is expressed in a metamodel. It is built by instantiating the constructs, i.e., metaclasses and associations, of the metamodel.

Definition 2 (Model). A model is defined by a tuple $\mathrm{M}=(\mathrm{M}, \mathrm{N}, \mathrm{A}, \tau)$ where:

- M is the metamodel in which the model is expressed.

$-\mathrm{N}$ is the set of metaclass instantiations of the metamodel $\mathrm{M}$, and $\mathrm{n} \in \mathrm{N}$ ranges over it. They are often simply referred to as instances when there is no possible confusion.

- $\mathrm{A} \subseteq \mathrm{N} \times(\mathrm{N} \times \mathbb{R})$ is the set of association instantiations of the metamodel $\mathrm{M}$, and $\mathrm{a} \in \mathrm{A}$ ranges over it. They are often referred to as links.

$-\tau$ is the typing function: $(\mathrm{N} \rightarrow \mathbb{N}) \cup(\mathrm{A} \rightarrow \mathbb{A})$. It records the type information of the instances and links in the model, i.e., from which metaclasses or associations of the metamodel $M$ they are instantiated.

\subsection{Model Conformance}

Not all models following the definitions above are valid, or "conform to" the metamodel: typing, multiplicity, and constraints need all to be respected.

Definition 3 (Model conformance). We say a model $\mathrm{M}=(\mathrm{M}, \mathrm{N}, \mathrm{A}, \tau)$ conforms to its metamodel $M$ or is valid when the following conditions are met:

1. type compatible:

$$
\forall \mathrm{a} \in \mathrm{A}, \tau(\mathrm{fst}(\mathrm{a})) \leq \mathrm{fst}(\mathrm{fst}(\tau(\mathrm{a}))) \text { and } \tau(\mathrm{fst}(\operatorname{snd}(\mathrm{a}))) \leq \mathrm{fst}(\operatorname{snd}(\tau(\mathrm{a})))
$$

Namely, the types of the link ends must be compatible with (being subtypes of) the types as specified in the corresponding association ends.

2. multiplicity compatible: $\forall \mathrm{n} \in \mathrm{N}, \mathbb{a} \in \mathbb{A}$,

if $\tau(\mathrm{n}) \leq \mathrm{fst}(\mathrm{fst}(\mathbb{a}))$,

then $\sharp\{\mathrm{a} \mid \mathrm{a} \in \mathrm{A}$ and $\tau(\mathrm{a})=\mathbb{a}$ and $\operatorname{fst}(\mathrm{a})=\mathrm{n}\} \in \operatorname{snd}(\operatorname{snd}(\mathbb{a}))$;

if $\tau(\mathrm{n}) \leq \mathrm{fst}(\operatorname{snd}(\mathbb{a}))$,

then $\sharp\{\mathrm{a} \mid \mathrm{a} \in \mathrm{A}$ and $\tau(\mathrm{a})=\mathbb{a}$ and $\mathrm{fst}(\operatorname{snd}(\mathrm{a}))=\mathrm{n}\} \in \operatorname{snd}(\mathrm{fst}(\mathbb{a}))$.

Namely, the number of link ends should conform to the specified multiplicity in the corresponding association end.

3. constraints hold: $\forall \mathbb{C} \in \mathbb{C}, \forall \mathrm{n} \in \mathrm{N}$ where $n$ is an instance of the context metaclass, i.e., $\tau(\mathrm{n}) \leq \mathrm{fst}(\mathbb{C})$, the boolean expression $\operatorname{snd}(\mathbb{C})$ should evaluate to true in model $\mathrm{M}$ for the contextual instance $\mathrm{n}$.

\section{Model Decomposition}

\subsection{Criteria}

Model decomposition starts from a model that conforms to a metamodel, and decomposes it into smaller parts. Our model decomposition technique is designed using the following as main criterion: the derived parts should be valid models conforming to the original metamodel. Achieving this goal has two main advantages: 
1. the derived parts, being themselves valid models, can be comprehended on their own according to the familiar abstract syntax and semantics (if defined) of the modeling language;

2. the derived parts can be wrapped up into modules and reused in the construction of other system models, following our modular model composition paradigm [6].

The decomposed smaller parts of a model are called its sub-models, formally defined below.

Definition 4 (Sub-model). We say a model $\mathrm{M}^{\prime}=\left(\mathbb{M}, \mathrm{N}^{\prime}, \mathrm{A}^{\prime}, \tau^{\prime}\right)$ is a sub-model of another model $\mathrm{M}=(\mathrm{M}, \mathrm{N}, \mathrm{A}, \tau)$ if and only if:

1. $\mathrm{N}^{\prime} \subseteq \mathrm{N}$;

2. $\mathrm{A}^{\prime} \subseteq \mathrm{A}$;

3. $\tau^{\prime}$ is a restriction of $\tau$ to $\mathrm{N}^{\prime}$ and $\mathrm{A}^{\prime}$.

In order to make the sub-model $\mathbf{M}^{\prime}$ also conform to $\mathbb{M}$, we will propose three conditions - one for the metamodel (Condition 3 below, regarding the nature of the constraints) and two conditions for the sub-model (Conditions 1 and 2). Altogether these three conditions will be sufficient to ensure conformance of the sub-model.

The starting point of our investigation is the definition of conformance (Definition (3). Three conditions must be met in order for sub-model $\mathrm{M}^{\prime}$ to conform to metamodel $\mathbb{M}$.

The first condition for conformance, type compatibility, follows directly from the fact that $\mathrm{M}^{\prime}$ is a sub-model of $\mathrm{M}$ and $\mathrm{M}$ conforms to $\mathrm{M}$. The second condition for conformance, which we call the multiplicity condition, concerns the multiplicities on the association ends in the metamodel $\mathbb{M}$. First the number of links ending at an instance of $\mathrm{M}^{\prime}$ must agree with the source cardinality of the corresponding association in the metamodel and second the number of links leaving an instance of $\mathrm{M}^{\prime}$ must agree with the target cardinality of the corresponding association.

To ensure the multiplicity condition for links ending at an instance of the sub-model, we will introduce the notion of fragmentable links, whose type (i.e., the corresponding association) has an un-constrained (i.e., being 0 ) lower bound for the source cardinality.

Definition 5 (Fragmentable link). Given a model $\mathrm{M}=(\mathbb{M}, \mathrm{N}, \mathrm{A}, \tau)$, a link $\mathrm{a} \in \mathrm{A}$ is fragmentable if $\operatorname{snd}(\mathrm{fst}(\tau(\mathrm{a})))=\left(0,{ }_{-}\right)$, where - represents any integer whose value is irrelevant for this definition.

Fragmentable incoming links of $\mathrm{M}$ to instances in $\mathrm{M}^{\prime}$ are safe to exclude but this is not the case for non-fragmentable links, which should all be included. We thus obtain the first condition on sub-model $\mathrm{M}^{\prime}$ :

Condition 1. $\forall a \in A$ where a is non-fragmentable, fst(snd(a)) $\in \mathrm{N}^{\prime}$ implies fst $(a) \in \mathrm{N}^{\prime}$ and $\mathrm{a} \in \mathrm{A}^{\prime}$. 
Let us now consider the multiplicity condition for links leaving an instance of $M^{\prime}$. To ensure this condition we shall require that $M^{\prime}$ includes all the links of $M$ that leave an instance of $\mathrm{M}^{\prime}$. In other words, there are in fact no links leaving $\mathrm{M}^{\prime}$. This is formally expressed in the following condition on the sub-model:

Condition 2. $\forall \mathrm{a} \in \mathrm{A}$, fst $(\mathrm{a}) \in \mathrm{N}^{\prime}$ implies $\mathrm{fst}(\operatorname{snd}(\mathrm{a})) \in \mathrm{N}^{\prime}$ and $\mathrm{a} \in \mathrm{A}^{\prime}$.

Conditions 1 and 2 together imply the multiplicity condition in the conformance definition.

The third condition in the conformance definition, which we call the constraint condition, requires that all metamodel constraints are satisfied in sub-model $\mathrm{M}^{\prime}$. To ensure this condition, we impose a restriction on the nature of the constraints. To this end, we introduce the notion of forward constraint.

Definition 6 (Forward constraint). A constraint $\mathbb{C}$ is forward if for any model $\mathrm{M}$ and any instance $\mathrm{n}$ of $\mathrm{M}$, the instances and links referenced by constraint $\mathbb{C}$ with contextual instance $\mathrm{n}$ are reachable from $\mathrm{n}$ in $\mathrm{M}$ (viewed as a directed graph).

We will only consider forward constraints in this paper. This is expressed in the following condition over the metamodel $M$ :

Condition 3. All constraints in metamodel M are forward constraints.

Note that we have formalized a core part of the EssentialOCL [7] in the companion technical report [5], which in principal excludes Alllnstances, called CoreOCL and we prove in [5] that all CoreOCL constraints are forward.

It is not difficult to see that both Conditions 2 and 3 imply the constraint condition in the conformance definition. Indeed Condition 2 implies that all instances and links reachable from an instance $\mathrm{n}$ in model $\mathrm{M}$ are also reachable in $\mathrm{M}^{\prime}$ if $\mathrm{M}^{\prime}$ does indeed contain $\mathrm{n}$. Condition 3 then implies that a constraint that is satisfied on contextual instance $\mathrm{n}$ in $\mathrm{M}$ is also satisfied in the sub-model $\mathrm{M}^{\prime}$ since it references the same instances and links in both models.

We thus obtain the following result:

Theorem 1. Given a metamodel $\mathrm{M}$, a model $\mathrm{M}$, and a sub-model $\mathrm{M}^{\prime}$ of $\mathrm{M}$, suppose that:

1. model $\mathrm{M}$ conforms to $\mathrm{M}$;

2. model $\mathrm{M}^{\prime}$ satisfies Condition 1 and 2 ;

3. metamodel M satisfies Condition [3;

then model $\mathrm{M}^{\prime}$ also conforms to the metamodel $\mathbf{M}$.

Proof. The result follows from the discussion above. 


\subsection{Algorithm}

From hereon we shall assume that the metamodel under consideration satisfies Condition 3. In this subsection we describe an algorithm that finds, for a given model $\mathrm{M}$, a decomposition of $\mathrm{M}$ such that any sub-model of $\mathrm{M}$ that satisfies both Condition 1 and 2 can be derived from the decomposition by uniting some components of the decomposition.

We reach the goal in two steps: (1) ensure Condition 1 and 2 with respect to only non-fragmentable links; (2) ensure again Condition 2 with respect to fragmentable links. (Condition 1 does not need to be re-assured because it only involves non-fragmentable links.) Details of each step are discussed below.

Treating instances as vertices and links as edges, models are just graphs. For illustration purpose, consider an example model as presented in Figure 1 where all fragmentable links are indicated by two short parallel lines crossing the links.

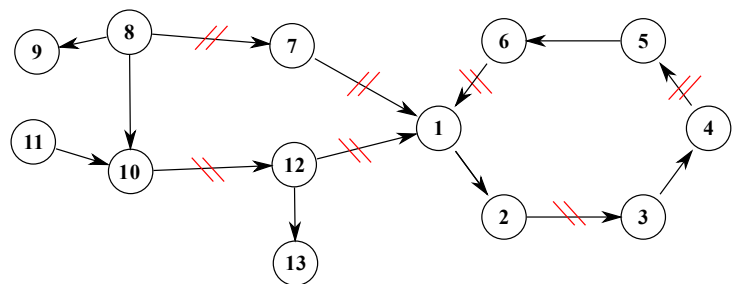

Fig. 1. An example model

Let $G$ be the graph derived by removing the fragmentable links from M. Because all the links in $G$ are non-fragmentable, for a sub-graph of $G$ to satisfy both Condition 11 and 2, an instance is included in the sub-graph if and only if all its ancestor and descendant instances are also included, and so are the links among these instances in $G$. These instances, from the point of view of graph theory, constitute a weakly connected component (wcc) of graph $G$ (i.e., a connected component if we ignore edge directions). The first step of the model decomposition computes all such wcc's of $G$, which disjointly cover all the instances in model $\mathrm{M}$, then puts back the fragmentable links. We collapse all the nodes that belong to one wcc into one node, (referred to as a wcc-node in contrast to the original nodes), and refer to the result as graph $W$. After the first step, the corresponding graph $W$ of the example model contains six wcc-nodes inter-connected by fragmentable links, as shown in Figure 2 .

The instances and links that are collapsed into one wcc-node in $W$ constitute a sub-model of $\mathrm{M}$ satisfying both Condition 11 and 2 , but only with respect to non-fragmentable links, because wcc's are computed in the context of $G$ where fragmentable links are removed. The second step of the model decomposition starts from graph $W$ and tries to satisfy Condition 2 with respect to fragmentable links, i.e., following outgoing fragmentable links. More specifically, we compute all the strongly connected components (scc's) in $W$ (see [10] for a definition of strongly connected components) and collapse all the nodes that belong to one 


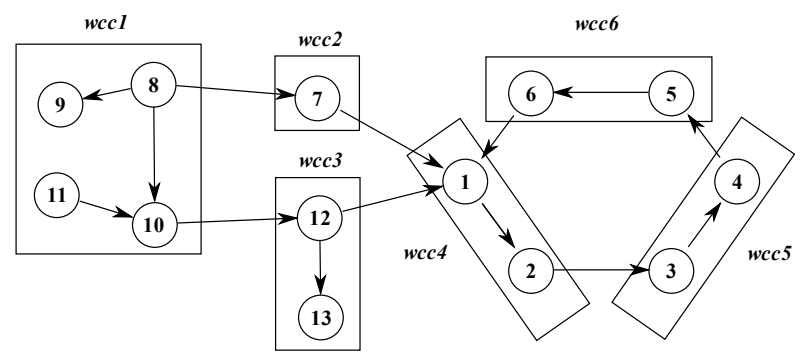

Fig. 2. The corresponding $W$ graph of the example model after step 1

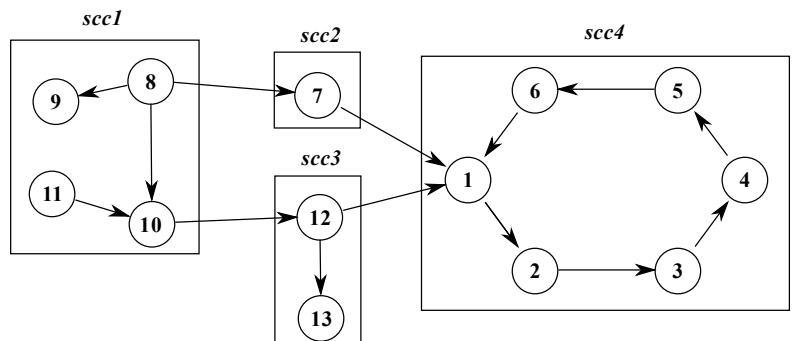

Fig. 3. The corresponding $D$ graph of the example model after step 2

scc into one node, (referred to as an scc-node), and refer to the result as graph $D$. After the second step, the corresponding graph $D$ of the example model looks like in Figure 3. The three wcc-nodes $w c c 4, w c c 5$ and $w c c 6$ of graph $W$ are collapsed into one scc-node scc4 because they lie on a (directed) cycle.

Note that we only collapse nodes of a strongly connected component in the second step instead of any reachable nodes following outgoing fragmentable links in $W$, because we do not want to loose any potential sub-model of $\mathrm{M}$ satisfying both Condition 1 and 2 on the way. More precisely, a set of nodes is collapsed only if for every sub-model $\mathrm{M}^{\prime}$ of $\mathrm{M}$ satisfying both Condition 11 and 2, it is either completely contained in $\mathrm{M}^{\prime}$ or disjoint with $\mathrm{M}^{\prime}$, i.e., no such $\mathrm{M}^{\prime}$ can tell the nodes in the set apart.

The computational complexity of the above algorithm is dominated by the complexity of computing weakly and strongly connected components in the model graph. Computing weakly connected components amounts to computing connected components if we ignore the direction of the edges. We can compute connected components and strongly connected components in linear time using depth-first search [10]. Thus the overall complexity is linear in the size of the model graph.

\subsection{Correctness}

Graph $D$ obtained at the end of the algorithm is a DAG (Directed Acyclic Graph) with all the edges being fragmentable links. Graph $D$ represents a decomposition 
of the original model $\mathrm{M}$ where all the instances and links that are collapsed into an scc-node in $D$ constitute a component in the decomposition. We call graph $D$ the decomposition hierarchy of model $\mathrm{M}$.

To relate the decomposition hierarchy to the sub-models, we introduce the concept of an antichain-node. An antichain-node is derived by collapsing a (possibly empty) antichain of scc-nodes (i.e., a set of scc-nodes that are neither descendants nor ancestors of one another, the concept of antichain being borrowed from order theory) plus their descendants (briefly an antichain plus descendants) in the decomposition hierarchy. To demonstrate the correctness of the algorithm, we prove the following theorem:

Theorem 2. Given a model $\mathrm{M}=(\mathrm{M}, \mathrm{N}, \mathrm{A}, \tau)$ and a sub-model $\mathrm{M}^{\prime}=\left(\mathrm{M}, \mathrm{N}^{\prime}, \mathrm{A}^{\prime}, \tau\right)$ of $\mathrm{M}, \mathrm{M}^{\prime}$ satisfies both Condition 1 and $[$ if and only if there exists a corresponding antichain-node of the decomposition hierarchy of $\mathrm{M}$ where $\mathrm{M}^{\prime}$ consists of the instances and links collapsed in this antichain-node.

Proof. We first demonstrate that if $\mathrm{M}^{\prime}$ consists of the set of instances and links that are collapsed in an antichain-node of the decomposition hierarchy of $\mathrm{M}$, then $\mathrm{M}^{\prime}$ satisfies both Condition 1 and 2 .

- Check $\mathrm{M}^{\prime}$ against Condition [1 given a non-fragmentable link a $\in A$, if fst(snd(a)) $\in \mathrm{N}^{\prime}$, we have $\mathrm{fst}(\mathrm{a}) \in \mathrm{N}^{\prime}$ because of the wcc computation in the first step of the model decomposition algorithm.

- Check $\mathrm{M}^{\prime}$ against Condition 2, given a non-fragmentable link a $\in \mathrm{A}$, if fst $(\mathrm{a}) \in$ $\mathrm{N}^{\prime}$, we have $\mathrm{fst}(\mathrm{snd}(\mathrm{a})) \in \mathrm{N}^{\prime}$ because of the wcc computation in the first step of the model decomposition algorithm. Given a fragmentable link $a \in A$, if fst $(a) \in N^{\prime}$, we have fst(snd $\left.(a)\right) \in N^{\prime}$ because of the scc computation in the second step of the model decomposition algorithm and because we take all the descendants into account.

We now demonstrate the other direction of the theorem, namely, if $\mathrm{M}^{\prime}$ satisfies both Condition 1 and 2, then there exists an antichain-node of the decomposition hierarchy of $\mathrm{M}$, such that $\mathrm{M}^{\prime}$ consists of the set of instances and links that are collapsed in this antichain-node.

We refer to the set of scc-nodes in the decomposition hierarchy where each includes at least one instance of $\mathrm{M}^{\prime}$ by $S$.

1. All the instances that are collapsed in an scc-node in $S$ belong to $\mathrm{M}^{\prime}$. Given an scc-node $s \in S$, there must exist an instance $\mathrm{n}$ collapsed in $s$ and $\mathrm{n} \in \mathrm{N}^{\prime}$ in order for $s$ to be included in $S$. Let $\mathrm{n}^{\prime}$ be another instance collapsed in $s$. Following the algorithm in Section 3.2 to compute the decomposition hierarchy, $n$ and $n^{\prime}$ are aggregated into one scc-node either in the first or the second step.

(a) If they are aggregated in the first step, that means the two instances are weakly connected by non-fragmentable links, and because $\mathrm{M}^{\prime}$ satisfies Condition 1 and 2, $\mathrm{n}^{\prime}$ should also be in $\mathrm{M}^{\prime}$.

(b) If they are aggregated in the second step but not in the first step, that means $\mathrm{n}$ and $\mathrm{n}^{\prime}$ are aggregated in two separate wcc-nodes in the first step, 
called $w$ and $w^{\prime}$, which are strongly connected by a path of fragmentable links. Referring to the other wcc-nodes on the path by $w_{1}, \ldots, w_{k}$, there exists a set of instances $\mathrm{n}_{0} \in w, \mathrm{n}_{0}^{\prime} \in w^{\prime}$, and $\mathrm{n}_{i}, \mathrm{n}_{i}^{\prime} \in w_{i}$ for $1 \leq i \leq k$, such that there are fragmentable links from $\mathrm{n}_{0}$ to $\mathrm{n}_{1}$, from $\mathrm{n}_{i}^{\prime}$ to $\mathrm{n}_{i+1}$ $(\forall i .1 \leq i<k)$ and from $\mathrm{n}_{k}^{\prime}$ to $\mathrm{n}_{0}^{\prime}$. Since $\mathrm{M}^{\prime}$ satisfies Condition 2 if the source vertex of these fragmentable links belongs to $\mathrm{M}^{\prime}$, so does the target vertex. Because the following pairs of instances: $\mathrm{n}$ and $\mathrm{n}_{0}, \mathrm{n}_{i}$ and $\mathrm{n}_{i}^{\prime}(\forall i .1 \leq i \leq k)$, and $\mathrm{n}_{0}^{\prime}$ and $\mathrm{n}^{\prime}$, are respectively collapsed in a wcc-node, if one vertex in a pair belongs to $\mathrm{M}^{\prime}$ then the other vertex in the pair must belong to $\mathrm{M}^{\prime}$ as well following Condition 1 and 2 . From the above discussion and by applying mathematical induction, $\mathrm{n}$ belonging to $\mathrm{M}^{\prime}$ implies that $\mathrm{n}^{\prime}$ belongs to $\mathrm{M}^{\prime}$ as well.

2. $S$ constitutes an antichain plus descendant. We partition $S$ into two subsets: $S_{1}$ contains all the scc-nodes in $S$ that do not have another scc-node also in $S$ as ancestor; $S_{2}$ contains the rest, i.e., $S_{2}=S \backslash S_{1}$. Clearly $S_{1}$ constitutes an antichain, and any scc-node in $S_{2}$ is a descendant of an scc-node in $S_{1}$ because otherwise the former scc-node should belong to $S_{1}$ instead of $S_{2}$. Moreover, $S_{2}$ contains all the descendants of scc-nodes in $S_{1}$. Given a child $s_{2}$ of an scc-node $s_{1} \in S_{1}$, the fragmentable link from $s_{1}$ to $s_{2}$ connects an instance $\mathrm{n}_{1}$ collapsed in $s_{1}$ to an instance $\mathrm{n}_{2}$ collapsed in $s_{2}$. Because $s_{1} \in S_{1}$, following the demonstrated item 1 above, we have $\mathrm{n}_{1} \in \mathrm{N}^{\prime}$. Because of the out-going fragmentable link from $\mathrm{n}_{1}$ to $\mathrm{n}_{2}$ and since $\mathrm{M}^{\prime}$ satisfies Condition 2 , we also have $\mathrm{n}_{2} \in \mathrm{N}^{\prime}$. Therefore we have $s_{2} \in S$. Furthermore, $s_{2} \notin S_{1}$ because it has $s_{1} \in S$ as its ancestor. Hence we have $s_{2} \in S_{2}$. Inductively we conclude that any descendant of $s_{1}$ belongs to $S_{2}$ and hence $S$ constitutes an antichain plus descendant.

3. Collapse all the scc-nodes in $S$ into an antichain-node called $A$. We demonstrate that $\mathrm{M}^{\prime}$ consists of the instances and links collapsed in $A$.

(a) Any instance of $\mathrm{M}^{\prime}$ is collapsed in $A$ because of the selection criteria of $S$, and any instance collapsed in $A$ is an instance of $\mathrm{M}^{\prime}$ following the demonstrated item 1 above. In other words, $\mathrm{M}^{\prime}$ and $A$ contain the same set of instances from $\mathrm{M}$.

(b) Because both $\mathrm{M}^{\prime}$ and $A$ span all the links in $\mathrm{M}$ that connect instances in them, $\mathrm{M}^{\prime}$ and $A$ also have the same set of links from $\mathrm{M}$.

\subsection{The Lattice of Sub-models}

Recall that a lattice is a partially-ordered set in which every pair of elements has a least upper bound and a greatest lower bound. Thanks to Theorem 2, we can now refer to a sub-model $\mathrm{M}^{\prime}$ of model $\mathrm{M}$ that satisfies both Condition 1 and 2 by the corresponding antichain-node $A$ in the decomposition hierarchy of M. Given a model $\mathrm{M}$, all the sub-models that satisfy both Condition 1 and 2 constitute a lattice ordered by the relation "is a sub-model of", referred to as the sub-model lattice of M. Let $A_{1}$ and $A_{2}$ denote two such sub-models. The least upper bound $\left(A_{1} \vee A_{2}\right)$ and the greatest lower bound $\left(A_{1} \wedge A_{2}\right)$ of $A_{1}$ and $A_{2}$ are computed in the following way: 


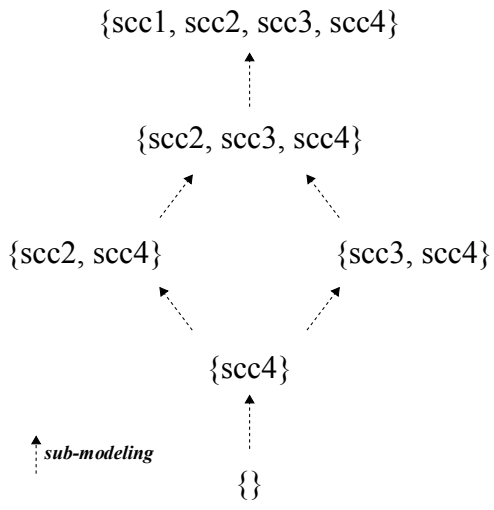

Fig. 4. The sub-model lattice of the example model in Figure 1 whose decomposition hierarchy is given in Figure 2

- $A_{1} \vee A_{2}$ is the antichain-node obtained by collapsing the scc-nodes of $A_{1}$ and $A_{2}$

- $A_{1} \wedge A_{2}$ is the antichain-node obtained by collapsing the common scc-nodes of $A_{1}$ and $A_{2}$.

The top of the sub-model lattice is $M$ itself, and the bottom is the empty sub-model.

For the example model discussed in Section 3.2 whose decomposition hierarchy is given in Figure 3, six possible antichain-nodes can be derived from the decomposition hierarchy, denoted by the set of scc-nodes that are collapsed. They are ordered in a lattice as shown in Figure 4.

\subsection{Implementation}

We have implemented the model decomposition technique 1]. The implementation takes a model of any metamodel that follows Definition 1 as input, and computes the decomposition hierarchy of it from which the sub-model lattice can be constructed by enumerating all the antichain-nodes of the decomposition hierarchy. Note that in the worst case where the decomposition hierarchy contains no edges, the size of the sub-model lattice equals the size of the power-set of the decomposition hierarchy, which is exponential.

\section{Application: Pruning Based Model Comprehension}

In this section, we demonstrate the power of our generic model decomposition technique by reporting one of its applications in a pruning-based model comprehension method. A typical comprehension question one would like to have answered for a large model is: 
Given a set of instances of interest in the model, how does one construct a substantially smaller sub-model that is relevant for the comprehension of these instances?

Model readers, when confronted with such a problem, would typically start from the interesting instances and browse through the whole model attempting to manually identify the relevant parts. Even with the best model documentation and the support of model browsing tools, such a task may still be too complicated to solve by hand, especially when the complexity of the original model is high. Moreover, guaranteeing by construction that the identified parts (together with the interesting instances) indeed constitute a valid model further complicates the problem.

Our model decomposition technique can be exploited to provide a linear time automated solution to the problem above. The idea is to simply take the union of all the scc-nodes, each of which contains at least one interesting instance, and their descendant scc-nodes in the decomposition hierarchy of the original model. We have implemented the idea in a Ecore [9] model comprehension tool [1] based on the implementation of the model decomposition technique.

To assess the applicability of the tool, a case study has been carried out. We have chosen the Ecore model of BPMN (Business Process Modeling Notation) [1] bpmn.ecore as an example, and one of BPMN's main concepts Gateway - for comprehension. Gateways are modeling elements in BPMN used to control how sequence flows interact as they converge and diverge within a business process. Five types of gateways are identified in order to cater to different types of sequence flow control semantics: exclusive, inclusive, parallel, complex, and event-based.

Inputs to the comprehension tool for the case study are the following:

- The BPMN Ecore model containing 134 classes (EClass instances), 252 properties (EReference instances), and 220 attributes (EAttribute instances). Altogether, it results in a very large class diagram that does not fit on a single page if one wants to be able to read the contents properly. Figure 5 shows the bird's eye view of this huge diagram.

- a set of interesting instances capturing the key notions of the design of gateways in BPMN: Gateway, ExclusiveGateway, InclusiveGateway, ParallelGateway, ComplexGateway, and EventBasedGateway.

After applying the tool, the result BPMN sub-model that contains all the selected interesting instances has only 17 classes, 7 properties, and 21 attributes. We observe that all the other independent concepts of BPMN such as Activity, Event, Connector, and Artifact, are pruned out. The class diagram view of the pruned BPMN model is shown in Figure 6. Note that it corresponds well to the class diagram that is sketched in the chapter for describing gateways in the BPMN 2.0 specification [11. We have also verified that the pruned BPMN model is indeed an Ecore instance by validating it against ecore.ecore in EMF 9 . 
Fig. 5. Bird's Eye View of the Class diagram of the BPMN Ecore model

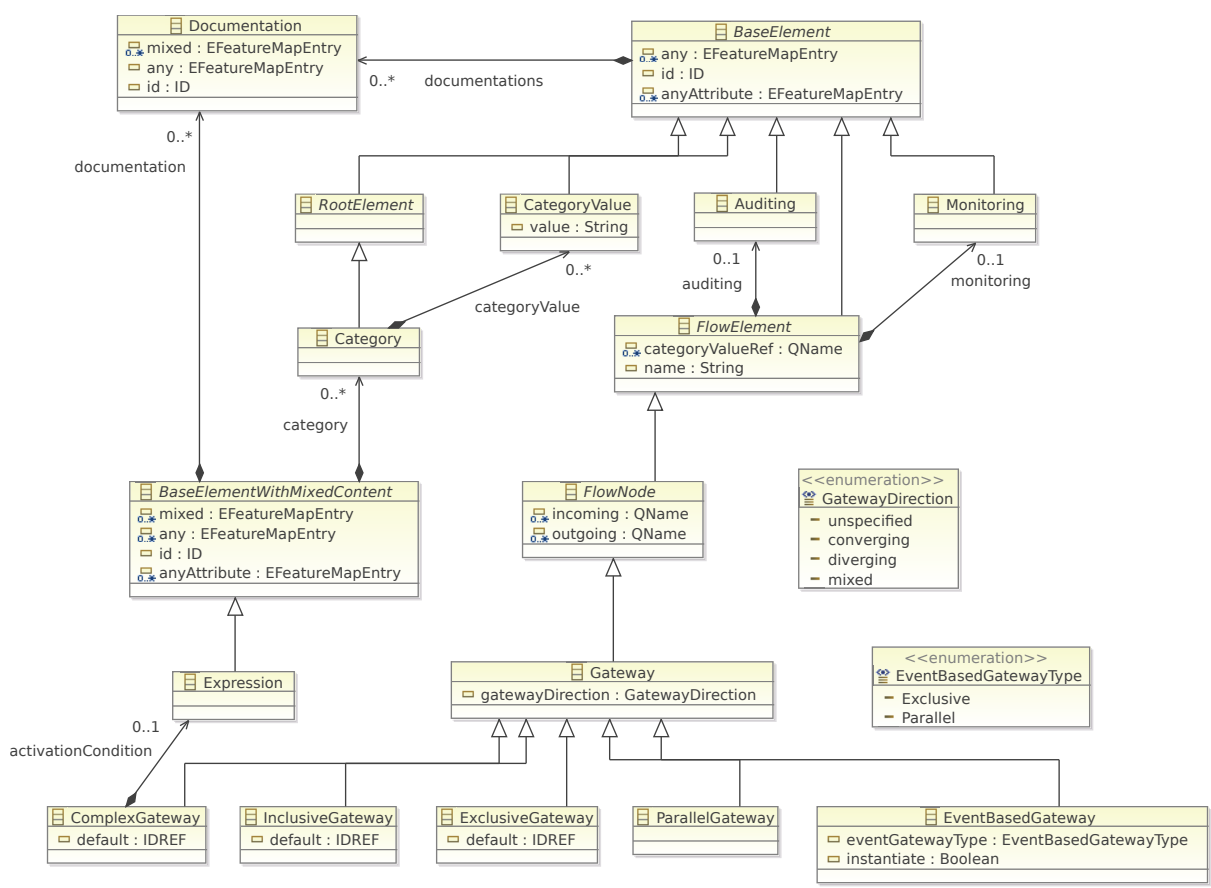

Fig. 6. Pruned class diagram for understanding the Gateway concept in BPMN

\section{Conclusion and Future Work}

The lattice of sub-models described in this paper should have applications beyond the application for model comprehension described in this paper. We foresee potential applications in the areas of model testing, debugging, and model reuse.

Given a software that takes models of a metamodel as input (e.g., a model transformation), an important part in testing the software is test case generation. Our model decomposition technique could help with the generation of new test 
cases by using one existing test case as the seed. New test cases of various complexity degree could be automatically generated following the sub-model lattice of the seed test case.

Moreover, our model decomposition can also help with the debugging activity when a failure of the software is observed on a test case. The idea is that we will find a sub-model of the original test case which is responsible for triggering the bug. Although both the reduced test case and the original one are relevant, the smaller test case is easier to understand and investigate.

A major obstacle to the massive model reuse in model-based software engineering is the cost of building a repository of reusable model components. A more effective alternative to creating those reusable model components from scratch is to discover them from existing system models. Sub-models of a system extracted by following our model decomposition technique are all guaranteed to be valid models hence can be wrapped up into modules and reused in the construction of other systems following our modular model composition paradigm [6].

Our model decomposition technique, described in this paper can be further improved: indeed it is currently based on three sufficient conditions that are not necessary. A consequence of this is that not all conformant sub-models are captured in the lattice of sub-models. A finer analysis of the constraints in the metamodel could result in weakening the three conditions and thus provide a more complete collection of conformant sub-models.

Acknowledgment. We would like to thank the anonymous referees for making numerous comments that helped us in improving the presentation of this paper.

\section{References}

1. Democles tool, http://democles.lassy.uni.lu/

2. Bae, J.H., Lee, K., Chae, H.S.: Modularization of the UML metamodel using model slicing. In: Fifth International Conference on Information Technology: New Generations, pp. 1253-1254 (2008)

3. Frantz, F.K.: A taxonomy of model abstraction techniques. In: Winter Simulation Conference, pp. 1413-1420 (1995)

4. Kagdi, H., Maletic, J.I., Sutton, A.: Context-free slicing of UML class models. In: ICSM 2005: Proceedings of the 21st IEEE International Conference on Software Maintenance, pp. 635-638. IEEE Computer Society, Washington, DC, USA (2005)

5. Kelsen, P., Ma, Q.: A generic model decomposition technique. Technical Report TR-LASSY-10-06, Laboratory for Advanced Software Systems, University of Luxembourg (2010), http://democles.lassy.uni.lu/documentation/TR_LASSY_10_06.pdf

6. Kelsen, P., Ma, Q.: A modular model composition technique. In: Rosenblum, D.S., Taentzer, G. (eds.) FASE 2010. LNCS, vol. 6013, pp. 173-187. Springer, Heidelberg (2010)

7. OMG. Object Constraint Language version 2.2 (February 2010) 
8. Sen, S., Moha, N., Baudry, B., Jézéquel, J.-M.: Meta-model pruning. In: Schürr, A., Selic, B. (eds.) MODELS 2009. LNCS, vol. 5795, pp. 32-46. Springer, Heidelberg (2009)

9. Steinberg, D., Budinsky, F., Paternostro, M., Merks, E.: EMF: Eclipse Modeling Framework, 2nd edn. Addison-Wesley Professional, Reading (2008)

10. Tarjan, R.E.: Depth-first search and linear graph algorithms. SIAM J. Comput. 1(2), 146-160 (1972)

11. White, S.A., Miers, D.: BPMN Modeling and Reference Guide. Future Strategies Inc. (2008) 